\title{
Seabed segmentation using optimized statistics of sonar textures
}

\author{
Imen Karoui ${ }^{1}$, Ronan Fablet ${ }^{1,2}$, Jean-Marc Boucher ${ }^{1}$ and Jean-Marie Augustin ${ }^{2}$ \\ ${ }^{1}$ Telecom Bretagne, UMR CNRS lab-STICC 3192, UEB, 29238 Brest Cedex 3, France \\ ${ }^{2}$ Ifremer, TSI-STH, Technopôle Brest Iroise, 29280 PLOUZANE, France
}

\begin{abstract}
We propose and compare two supervised algorithms of the segmentation of textured sonar images with respect to seafloor types. We characterize sea-floors by a set of empirical distributions estimated on texture responses for a wide set of different filters with various parameterizations and we introduce a novel similarity measure between sonar textures in this feature space. Our similarity measure is defined as a weighted sum of Kullback-Leibler divergences between texture features. The weight setting is twofold. First each filter is weighted according to its discrimination power: the computation of these weights are issued from a margin maximization criterion. Second, an additional weight, evaluated as an angular distance between the incidence angles of the compared texture samples, is considered to take into account sonar image acquisition process that leads to a variability of the backscattered (BS) value and of the texture aspect with the incidence angle range. A Bayesian framework is used in the first algorithm where the conditional likelihoods are expressed using the proposed similarity measure between local pixel statistics and the seafloor prototype statistics. The second method is based in a variational framework as the minimization of a region-based functional that involves the similarity between global region texture based statistics and the predefined prototypes.
\end{abstract}

\section{Index Terms}

Texture, sonar images, feature selection, angular backscattering, segmentation, MMP, active regions, level sets.

\section{INTRODUCTION}

Acoustic remote sensing, such as high-resolution multibeam and sidescan sonars, provides new means for in-situ observation of the seabed. The characterization of these high-resolution sonar images is important for a number of practical applications such as marine geology, commercial fishing, offshore oil prospecting and drilling [1], [2], [46], [47].

The segmentation and the classification of sonar images with respect to seafloor types (rocks, mud, sand,...) is the key goal behind the analysis of these acoustic images. This task raises however two major difficulties. The first task is to deal with texture in these images. Previous methods are generally based only on backscattered (BS) intensity models and several parametric families of probabilistic distribution functions have been suggested (Rayleigh distribution, K distribution, Weibull distribution etc) [5], [6], [44]-[47]. These first order statistics are not sufficient when high-resolution sonar images involve textures, which is the case of most sonar images (Fig.1). 


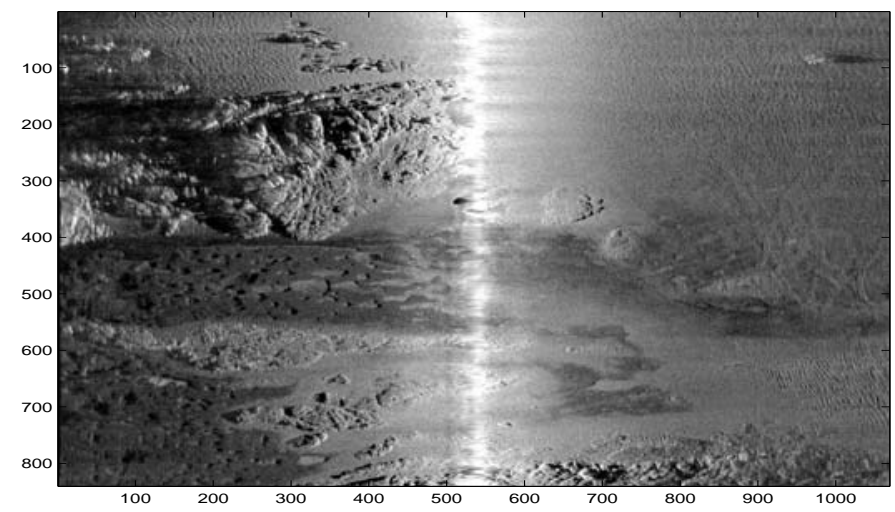

Fig. 1. A typical sidscan sonar image, (Rebent, Ifremer)

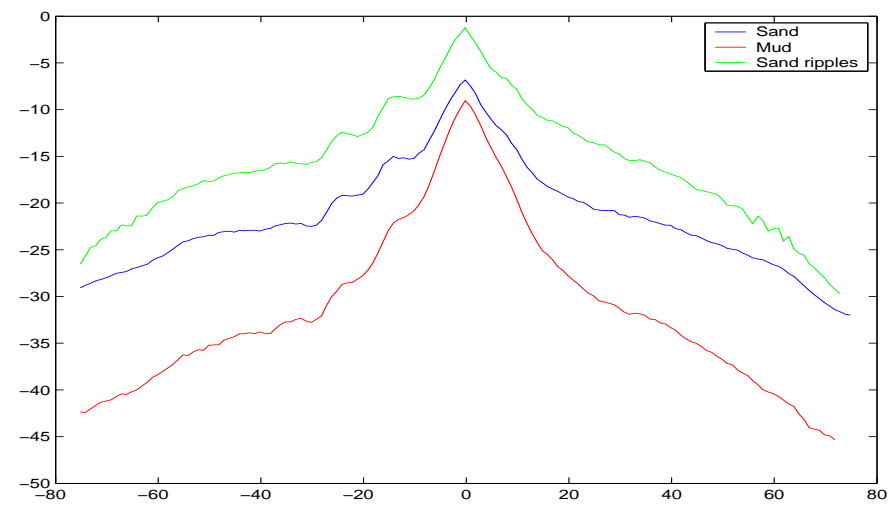

Fig. 2. BS evolution with the incidence angles for the three seafloor types of Fig.1: sand, mud and sand ripples

The other important issue arising in seabed texture characterization is a built-in feature of sonar observation: the value of BS measure depends both on the seafloor type and on the incident angle of the reflected acoustic signal, ranging typically from $-85^{\circ}$ to $+85^{\circ}$. Figure 2 shows the BS evolution for three different seafloor types : sand, sand ripples and mud. In addition to the BS variability within incidence angles, seafloor textures are dependent on the incidence angles. Figures 3 and 4 show a sonar image composed by sand ripples and rocks respectively for two angular sectors: $\left[80^{\circ}, 85^{\circ}\right]$ and $\left[5^{\circ}, 40^{\circ}\right]$. The texture of sand ripples shows a loss of contrast in the specular sector $\left[5^{\circ}, 40^{\circ}\right]$ : the steep grazing angle reduces the backscatter differences between facing and trailing slopes, while at low incidence angles much of the variation is lost due to increasing sonar shadow. A similar loss of contrast is observable in the rock samples (Fig.4). BS behavior according to the incidence angles has been of wide interest for sonar imaging [9]-[12], [46]. Parametric and non parametric techniques have been proposed to model sonar image behavior with respect to the incidence angle variations. The effect of the incidence angle on the BS has also been explored as a discriminating feature for seafloor recognition [13], [14], [47]. However no studies have proposed a method to accurately compensate this phenomena because of the joint dependency of the seafloor types and on the local bathymetry which is generally unknown for sidescan sonar images. To our knowledge, the effects of the 

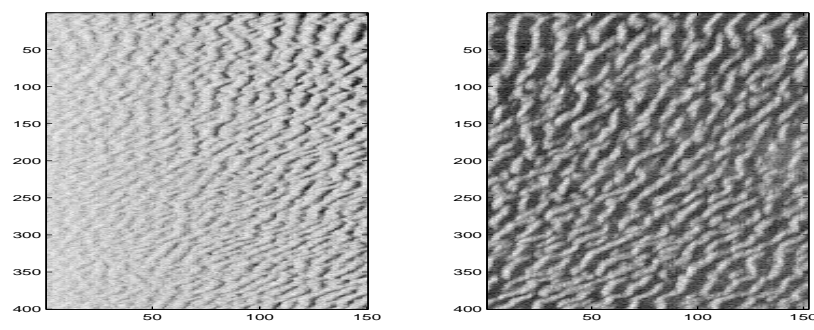

Fig. 3. Sonar image composed of sand ripples, Left $\left[5^{\circ}, 40^{\circ}\right]$ and right $\left[80^{\circ}, 85^{\circ}\right]$.
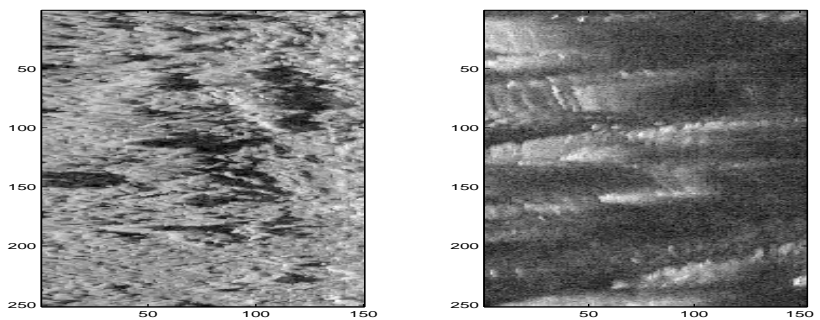

Fig. 4. Rock texture for two angular sectors. Left $\left[5^{\circ}, 40^{\circ}\right]$ and right $\left[80^{\circ}, 85^{\circ}\right]$.

incidence angle on textured seabed features have not been addressed for segmentation issues. Only some studies were interested in simulating the behavior of oriented and textured seafloor types [15]-[18]. These methods are mainly based on shape-from-shading [19] and were restricted to synthetic images or to real sonar images involving only one seafloor type.

In this work, we aim at using texture information within sonar seabed images, and at developing segmentation algorithms that take into account angular variability of BS and textural features. We propose to characterize seafloors by a wide set of marginal distributions of their filter responses and we measure seafloor similarities according to a weighted sum of Kullback-Leibler divergences [28] in this feature space. To cope with seafloor angular dependency, we introduce an additional weighting factor, evaluated as an angular distance between the compared texture samples: this angular distance is measured according to Gaussian kernels, whose variance sets the level of the angular variability depending on textures and sea-floor types. The proposed incidence-angle-and-texture based similarity measure is exploited to develop two different segmentation schemes. We first state the segmentation issue as a Bayesian pixel-based labeling according to local texture features. The second approach relies on a region-level variational framework, which resorts to a level-set minimization of an energy criterion involving global region-based seafloor statistics.

The paper is organized as follows. The seafloor similarity measure is introduced in Section II. The Bayesian segmentation method is detailed in Section III. The region based segmentation algorithm is described in Section IV. Experiments are reported and discussed in Section V and conclusions are drawn in Section VI. 

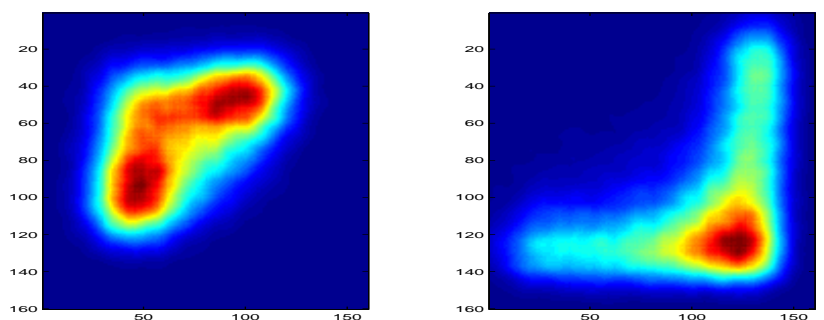

Fig. 5. Co-occurrence distributions computed for parameters $(d, \theta)=\left(6,0^{\circ}\right)$. Left $\left[5^{\circ}, 40^{\circ}\right]$ and right $\left[80^{\circ}, 85^{\circ}\right]$.

\section{SONAR TEXTURE SIMILARITY MEASURE}

Texture based segmentation of seabed sonar images generally relies on Haralick [30] parameters or scalar spectral and filter coefficients to model textures [20]-[22]. Recently, in the field of texture analysis, features computed as statistics of local filter responses have been shown to be relevant and discriminant texture descriptors [23]-[27]. Motivated by these studies, we propose to use texture features computed as marginal distributions of a wide set of filter seafloor responses. Each seafloor type denoted by $T_{k}$ is characterized by a set $Q_{k}$ composed of the marginal distributions of the seafloor with respect to the predefined filters. The following is issued:

- 121 co-occurrence distributions with horizontal and vertical displacements denoted by $d_{x}$ and $d_{y}$ respectively $\left(d_{x}, d_{y}\right) \in\{0,1, \ldots, 10\}$.

- 50 distributions of the magnitude of Gabor filter responses, computed for combination of parameters $\left(f_{0}, \sigma, \theta\right)$ where $f_{0}$ is the radial frequency, $\sigma$ is the standard deviation and $\theta$ the orientation, such that $f_{0} \in\left\{\frac{\sqrt{2}}{2^{k}}\right\}_{k=1: 6}$, $\sigma \in\left\{2^{k} \sqrt{2}\right\}_{k=2: 5}$ and $\theta \in\left\{0^{\circ}, 25^{\circ}, 45^{\circ}, 90^{\circ}, 135^{\circ}\right\}$.

- 48 distributions of the energy of the image wavelet packet coefficient computed for different bands (we used three wavelet types: Haar, Daubechies and Coiflet).

Sonar texture variability with respect to the incidence angles induces a variability in texture features. Figures 5 and 6 show the co-occurrence matrices of the images involving sand ripples and rocks displayed on figures 3 and 4 respectively. A change in co-occurrence distributions can be noticed. In the angular sector $\left[5^{\circ}, 40^{\circ}\right]$, the co-occurrence is bimodal due to the alternation of dark and light values in the image of sand ripples, whereas in the sector $\left[80^{\circ}, 85^{\circ}\right]$, the loss of contrast between dark and light values in the image of sand ripples leads to unimodal distribution. For co-occurrence distributions related to rock samples, a change in the variance is depicted. To deal with this problem, we propose to define angular subdomains, in which texture characteristics can be regarded as homogeneous. Each angular sector is indexed by $j$ and is characterized by a mean incidence angle value $\Theta_{j}$. Formally, this leads to introducing a state variable $z_{s}$ assigning pixel $s$ to a given angular subdomain. $\left(\theta_{s}, z_{s}\right)$ where $\theta_{s}$ is the incidence angle of pixel $s$, is then modeled as a Gaussian mixture, and the assignment likelihood $p\left(\Theta_{j} / z_{s}\right)$ is given by:

$$
p\left(\Theta_{j} / z_{s}\right)=\frac{\exp \left(\frac{-\left(\Theta_{j}-\theta_{s}\right)^{2}}{\sigma_{j}^{2}}\right)}{\sum_{i=1}^{J} \exp \left(\frac{-\left(\Theta_{i}-\theta_{s}\right)^{2}}{\sigma_{i}^{2}}\right)}
$$



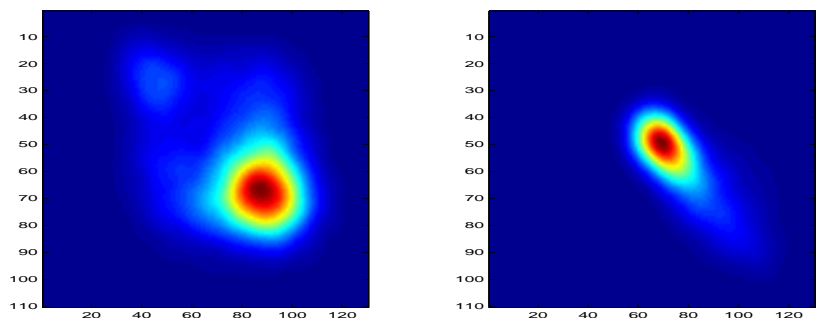

Fig. 6. Co-occurrence distributions computed for parameters $(d, \theta)=\left(6^{\circ}, 0\right)$. Left $\left[5^{\circ}, 40^{\circ}\right]$ and right $\left[80^{\circ}, 85^{\circ}\right]$.

Hence, the assignment of a given region $R$ to an angular subdomain is given by:

$$
\pi_{R}(j)=\frac{1}{|R|} \int_{s} p\left(\Theta_{j} / z_{s}\right) d s
$$

The angular bounds, defined by the parameters $\Theta_{j}$ and $\sigma_{j}$ are set experimentally and each seafloor type is characterized by $Q_{k}=\left\{Q_{f, j}\left(T_{k}\right)\right\}_{f=1: F, j=1: J}$, its filter responses estimated on the $J$ angular domains. Note that $f$ accounts both for filter types and various associated parameterizations: $f=1 \rightarrow 121$ refers to co-occurrence distributions, $f=122 \rightarrow 171$ to Gabor filter and $f=172 \rightarrow 219$ to wavelet based distributions. These statistics are computed using Parzen window estimation [29]. Formally, $Q_{f, j}\left(T_{k}, \alpha\right)=\frac{1}{\pi_{T_{k}}(j)} \int_{T_{k}} p\left(\Theta_{j} / z_{s}\right) g_{\sigma_{f}}\left(h_{f}(s)-\alpha\right) d s$, where $h_{f}$ is the filter response indexed by $f$ (for a co-occurrence matrix with parameters $\mu=(d, \theta), h_{f}: \Omega \rightarrow[1, N g] \times[1, N g]$ and $h_{f}(s)=(I(s), I(s+\mu))$ where $I(s)$ is the gray value at pixel $s$ and $N g$ the total gray level number) $g_{\sigma_{f}}$ a Gaussian kernel with zero mean and standard deviation $\sigma_{f}$.

We define the similarity measure between a texture sample $T$ with feature set $D(T)=\left\{D_{f, j}(T)\right\}_{f=1: F, j=1: J}$ and a given seafloor type $T_{k}$ as follows:

$$
K L_{w}^{\Theta}\left(Q^{k}, D(T)\right)=\sum_{j=1}^{J} \sum_{f=1}^{F} \pi_{T}(j) w_{f}^{2} K L\left(Q_{f, j}^{k}, D_{f, j}(T)\right), \sum_{f=1}^{F} w_{f}^{2}=1 .
$$

where $D_{f, j}(T)$ is the distribution related to filter $f$ and estimated on region $T$ for angular sector $j: D_{f, j}(T, \alpha)=$ $\frac{1}{\pi_{T}(j)} \int_{T} p\left(\Theta_{j} / z_{s}\right) g_{\sigma_{f}}\left(h_{f}(s)-\alpha\right) d s,\left\{w_{f}^{2}\right\}_{f=1: F}$ are the feature weights, $K L$ denotes for the Kullback-Leibler divergence [28]; for two probability distributions $Q$ and $D, K L(Q, D)=\int Q(\alpha) \log \left(\frac{Q(\alpha)}{D(\alpha)}\right) d \alpha$.

The resulting weighting factors $\left\{w_{f}^{2}\right\}_{f=1: F}$ are exploited on one hand for filter selection to keep only the distributions corresponding to the significant weights, and, on other hand, for the definition of an optimized texturebased similarity measure $K L_{w}^{\Theta}$ given the selected distributions. In a supervised context, the weights are estimated from a training set $\mathcal{T}$ composed of $N$ labeled texture samples: $\mathcal{T}=\left\{\left(D(T), s_{T}\right)\right\}$ where $s_{T}$ is the class of sample $T$. Formally, $\left\{w_{f}^{2}\right\}_{f=1: F}$ are issued from the maximization of the global margin defined as follows:

$$
M_{w}^{\mathcal{T}}=\sum_{T \in \mathcal{T}} M_{w}(T)
$$

where

$$
M_{w}(T)=K L_{w}^{\Theta}\left(Q^{d_{T}}, D(T)\right)-K L_{w}^{\Theta}\left(Q^{s_{T}}, D(T)\right)
$$


where $d_{T}$ is the nearest class to $T$ different from $s_{T}$ according to the similarity measure $K L_{w}^{\Theta}$ :

$$
d_{T}=\arg \min _{k \neq s_{T}} K L_{w}^{\Theta}\left(Q^{k}, D(T)\right)
$$

The maximization of the margin criterion $M_{w}^{\mathcal{T}}$ is carried out using a stochastic gradient method as detailed in our previous work [48].

\section{BAYESIAN SONAR IMAGE SEGMENTATION}

As far as bayesian image segmentation is concerned, the most popular criteria are the Maximum A Posteriori (MAP) [42] and the Maximum Marginal Probabiility (MMP) [31]. It has been shown that the MMP estimation criterion is more appropriate for image segmentation than the MAP criterion [31]. The MAP estimate assigns the same cost to every incorrect segmentation regardless the number of pixels at which the estimated segmentation differs from the true one, whereas the MMP algorithm minimizes the expected value of the number of misclassified pixels. As shown in [31], the MMP procedure is equivalent to maximizing the marginal of the class labels. Let us introduce the following notations:

- $S$ the image lattice composed of $N$ pixels;

- $X=\left\{x_{s}\right\}_{s \in S}$ the label random field;

- $Y=\left\{y_{s}\right\}_{s \in S}$ the random field of the observations, the textural feature in our case.

Formally, the MMP scheme is equivalent to the maximization of:

$$
\hat{x}_{s}^{M M P}=\arg \max _{k \in\{1: K\}} p\left(x_{s}=k / y\right)
$$

In general, pixel conditional likelihood are computed according to local texture features like Gabor or wavelet coefficients. Here, we aim at using the proposed similarity measure $K L_{w}^{\Theta}$. We associate to each pixel $s$ a set of features $D\left(W_{s}\right)=\left\{D_{f}\left(W_{s}\right)\right\}_{f=1: F}$ estimated according to a Parzen estimation method [29], within a square window $W_{s}$ centered at $s$. The window size that we denote by $T_{W}$ is set by the user according to texture coarseness. In our case, the observation denoted by a random field $Y$ is specified by $\left\{y_{s}=D\left(W_{s}\right)\right\}_{s \in S}$. The conditional likelihood at each pixel $s$ with respect to class $k$ is then defined from the similarity measure $K L_{w}^{\Theta}$ by:

$$
p\left(y_{s} / x_{s}=k\right)=\frac{\exp ^{-K L_{w}^{\ominus}\left(Q^{k}, D\left(W_{s}\right)\right)}}{\sum_{i=1}^{K} \exp ^{-K L_{w}^{\Theta}\left(Q^{i}, D\left(W_{s}\right)\right)}}
$$

As a prior $P_{X}$, we consider a Markov random field associated to an 8-neighborhood system with potential functions given by:

$$
U_{2}(x)=\sum_{s \in S} \sum_{t \in c_{s}} \alpha_{c}\left(1-\delta\left(x_{s}, x_{t}\right)\right)
$$

where $\delta$ is the delta function, $\alpha_{c} \in\left\{\alpha_{H}, \alpha_{V}, \alpha_{D}\right\}$ are real parameters assigned respectively to horizontal, vertical and diagonal cliques.These parameters are estimated using the ICE (Iterative Conditional Estimation) procedure [43]. Using Bayes rule the posterior distribution is expressed as follows:

$$
p(X, Y)=p(Y / X) p(X)=p(X) \prod_{s} p\left(y_{s} / x_{s}\right) \approx \exp \left(\sum_{s \in S} \sum_{t \in c_{s}}-\alpha_{c}\left(1-\delta\left(x_{s}, x_{t}\right)\right)+\log \left(p\left(y_{s} / x_{s}\right)\right)\right)
$$


The maximization of local probabilities $p\left(x_{s}=k / y\right)$ is carried out as follows [31]: we use the Gibbs sampler to generate a discrete-time Markov chain $X(t)$ which converges in distribution to a random field with probability mass functions $P(X / Y)$. The marginal conditional distributions $p\left(x_{s}=k / y\right)$ are then approximated as the fraction of time the Markov chain spends in state $k$ for each class $k$ and each pixel $s$. The MMP segmentation steps are the following:

- Simulation of $T_{\max }$ realizations of $x_{1}, x_{2}, \ldots, x_{T_{\max }}$ of $X$ using Gibbs sampler;

- Using the realizations $x_{1}, x_{2}, \ldots, x_{T_{\max }}, p\left(x_{s} / y\right)$ is estimated using the the frequency of each realizations:

$$
p\left(x_{s}=k / y\right)=\frac{\delta\left(x_{s}^{1}-k\right)+\ldots+\delta\left(x_{s}^{1}-k\right)}{T_{\max }} ;
$$

- Choose as $x_{s}$ the class that maximizes $p\left(x_{s}=k / y\right)$.

\section{VARIATIONAL SONAR IMAGE SEGMENTATION}

Unlike the Bayesian scheme, the second approach is stated at a region level as the minimization of a constrained energy criterion $E\left(\left\{\Omega_{k}\right\}_{k=1: K}\right)=E_{1}+E_{2}$, where $\Omega_{k}$ is the domain composed of all pixels attributed to the class $k, E_{1}$ is a texture-based data-driven term and $E_{2}$ a regularization term as detailed below.

\section{A. Functional terms}

$E^{1}\left(\left\{\Omega_{k}\right\}_{k=1: K}\right)$ is evaluated as the log-likelihood of a given partition with respect to texture models. It is evaluated as the sum of the similarities according to the measure $K L_{w}^{\Theta}$ between each region $\Omega_{k}$ and its corresponding class $T_{k}$ :

$$
E_{1}\left(\left\{\Omega_{k}\right\}_{k=1: K}\right)=\sum_{k=1}^{K} K L_{w}^{\Theta}\left(Q^{k}, D\left(\Omega_{k}\right)\right)
$$

where $D_{f, j}\left(\Omega_{k}\right)$ is the marginal distribution of the image response to the filter indexed by $f$. For angular domain $j, D_{f, j}\left(\Omega_{k}\right)$ is estimated according to Parzen method [29].

$E_{2}$ is a regularization term, it penalizes the lengths of region contours and is expressed by:

$$
E_{2}=\sum_{k=1}^{K} \gamma_{k}\left|\Gamma_{k}\right|, \gamma_{k} \in \Re_{+}
$$

where $\left|\Gamma_{k}\right|$ the length of the contour $\Gamma_{k}$ associated to the region $\Omega_{k}$.

\section{B. Computation of the evolution equation}

We solve for the minimization of the functional $E$ using a gradient descent technique. It relies on the computation of the first derivative of $E$ according to regions $\left\{\Omega_{k}\right\}_{k=1: K}$. The evolution equation of region contours $\left\{\Gamma_{k}\right\}_{k=1: K}$ is then given by the following dynamic scheme [32]:

$$
\left\{\begin{array}{l}
\frac{\partial \Gamma_{k}(x, t)}{\partial t}=F_{k}(x, t) \vec{N}_{k} \\
\Gamma_{k}(x, 0)=\Gamma_{k}^{0}
\end{array}\right.
$$

where $\vec{N}_{k}$ is the unit inward normal to $\Gamma_{k}$ at pixel $x$ and at time $t$ and $F_{k}$ the velocity field (in our case $F_{k}=\nabla E_{k}$, the derivative of $E$ with respect to $\Gamma_{k}$ ). 
The explicit implementation of the curve evolution according to the latter dynamic scheme using a difference approximation scheme can not deal with topological changes of the moving front. This could be avoided by introducing the level set method proposed by Osher and Sethian [33]. The basic idea of the method is the implicit representation of the moving interface $\Gamma$ by a higher dimensional hypersurface $\varphi$ (the level set function) such that the zero level set of $\varphi$ is actually the set of $\Gamma$ and:

$$
\left\{\begin{array}{l}
\Omega_{\text {inside }}=\{s \in \Omega / \varphi(s)>0\} \\
\Omega_{\text {outside }}=\{s \in \Omega / \varphi(s)<0\}
\end{array}\right.
$$

The evolution of the contours $\left\{\Gamma_{k}\right\}_{k=1: K}$ (equation 13) is then equivalent to the evolution of the level set functions $\left\{\varphi_{k}\right\}_{k=1: K}[33]:$

$$
\frac{\partial \varphi_{k}(s, t)}{\partial t}=F_{k}(s, t)\left|\nabla \varphi_{k}(s, t)\right|, \forall s \in \Omega_{k}
$$

$E_{2}$ can be expressed using level set functions $\varphi_{k}[36]$ :

$$
E_{2}=\sum_{k=1}^{K} \gamma_{k} \lim _{\alpha \rightarrow 0} \int_{\Omega} \delta_{\alpha}\left(\varphi_{k}\right)\left|\nabla \varphi_{k}\right| d s .
$$

where $\delta_{\alpha}$ is a regularized delta function:

$$
\delta_{\alpha}(s)=\left\{\begin{array}{l}
\frac{1}{2 \alpha}\left(1+\cos \left(\frac{\pi s}{\alpha}\right)\right) \text { if }|s| \leq \alpha \\
0 \text { if }|s|<\alpha
\end{array}\right.
$$

In order to cope with multi-class segmentation and to fulfill the image partition constraint, we use an additional term $E_{3}$ given by the following functional [34]:

$$
E_{3}=\frac{\lambda}{2} \int_{\Omega}\left(\sum_{k=1}^{K} H_{\alpha}\left(\varphi_{k}\right)-1\right)^{2} d s, \lambda \in \Re^{+}
$$

where $H_{\alpha}$ is a regularized Heaviside function:

$$
H_{\alpha}(s)=\left\{\begin{array}{l}
\frac{1}{2}\left(1+\frac{s}{\alpha}+\frac{1}{\pi} \sin \left(\frac{\pi s}{\alpha}\right)\right) \text { if }|s| \leq \alpha \\
1 \text { if } s>\alpha \\
0 \text { if } s<-\alpha
\end{array}\right.
$$

As $E=E_{1}+E_{2}+E_{3}$, we have $\frac{\partial \varphi_{k}}{\partial t}(s, t)=\frac{\partial \varphi_{k}^{1}}{\partial t}(s, t)+\frac{\partial \varphi_{k}^{2}}{\partial t}(s, t)+\frac{\partial \varphi_{k}^{3}}{\partial t}(s, t)$ where $\frac{\partial \varphi_{k}^{i}}{\partial t}, i=1,2,3$ are the evolution equation terms associated respectively to functionals $E_{i}, i=1,2,3$.

The derivatives of the energy terms $E_{2}$ and $E_{3}$ are directly estimated from level set functions [35], [36].

$$
\begin{gathered}
\frac{\partial \varphi_{k}^{2}}{\partial t}(s, t)=\gamma_{k} \delta_{\alpha}\left(\varphi_{k}\right) \operatorname{div}\left(\frac{\nabla \varphi_{k}}{\left|\nabla \varphi_{k}\right|}\right), \forall k \in\{1: K\} . \\
\frac{\partial \varphi_{k}^{3}}{\partial t}(s, t)=-\delta_{\alpha}\left(\varphi_{k}\right) \lambda\left(\sum_{k=1}^{K}\left(H_{\alpha}\left(\varphi_{k}\right)-1\right)\right), \forall k \in\{1: K\} .
\end{gathered}
$$

The evolution equation related to $E_{1}$ is more complex, since it involves computations over the spatial support of each region. To differentiate $E_{1}$, we use shape derivative tools, especially the Gâteaux derivative theorem given in 
[37]. As detailed in Annex A, it leads to:

$$
\frac{\partial \varphi_{k}^{1}}{\partial t}(s, t)=-\sum_{j=1}^{J} \sum_{f=1}^{F} w_{f}^{2} p\left(\Theta_{j} / z_{s}\right)\left[K L\left(Q_{f, j}^{k}, D_{f, j}^{k}\right)-\left(\frac{Q_{f, j}^{k}}{D_{f, j}} * g_{\sigma_{f}}\left(h_{f}(s)\right)-1\right)\right]\left|\nabla \varphi_{k}\right|
$$

where $*$ is the convolution symbol.

The evolution equation related to the energy $E_{1}$ (equation (21)) is composed of two terms:

- A global term $-\sum_{j=1}^{J} \sum_{f=1}^{F} w_{f}^{2} p\left(\Theta_{j} / z_{s}\right) K L\left(Q_{f, j}^{k}, D_{f, j}\right)$ : this term is always negative or null. It is a contraction force that reduces the size of heterogeneous regions;

- A local term $\sum_{j=1}^{J} \sum_{f=1}^{F} w_{f}^{2} p\left(\Theta_{j} / z_{s}\right)\left(\frac{Q_{f, j}^{k}}{D_{f, j}}-1\right) * g_{\sigma_{f}}\left(h_{f}(s)\right)$ : this term locally compares the features values at each pixel. This term can be positive or negative and aims at readjusting the statistics inside the regions $D_{f, j}\left(\Omega_{k}\right)$ to fit to prototype models $Q_{f, j}^{k}$. The contribution of each descriptors $f$ is weighted by $w_{f}^{2}$ and $p\left(\Theta_{j} / z_{s}\right)$, the relative contribution of descriptor $f$ and of angular sector $j$.

The overall evolution equations of the contours $\left\{\Gamma_{k}\right\}_{k=1: K}$ are the following:

$$
\begin{aligned}
& \frac{\partial \varphi_{k}}{\partial t}(s, t)=-\delta_{\alpha}\left(\varphi_{k}\right)\left[\sum_{j=1}^{J} \sum_{f=1}^{F} w_{f}^{2} p\left(\Theta_{j} / z_{s}\right)\left(K L\left(Q_{f, j}^{k}, D_{f, j}\right)-\frac{Q_{f, j}^{k}}{D_{f, j}} * g_{\sigma_{f}}\left(h_{f}(s)\right)+1\right)+\right. \\
& \left.\gamma_{k} \operatorname{div}\left(\frac{\nabla \varphi_{k}}{\left|\nabla \varphi_{k}\right|}\right)-\lambda\left(\sum_{k=1}^{K}\left(H_{\alpha}\left(\varphi_{k}\right)-1\right)\right)\right], \forall k \in\{1, \ldots, K\}
\end{aligned}
$$

We apply these coupled evolution equations until convergence.

\section{EXPERIMENTAL RESULTS AND DISCUSSION}

In previous work, we have tested the method on various optic textures (Brodatz textures). The method was compared to other texture classification methods and some results are reported in [48], [49]. Here, we evaluate the proposed seabed segmentation technique for different real sonar images acquired by a sidescan sonar, as part of a natural seabed mapping project (IFREMER, Rebent Project) [41]. A reference interpretation by an expert is available [41]. Figure 7 shows the set of images on which we carried out the experiments. We superimposed on these images the manual expert segmentation. Image I1 is composed of three seafloor types: rock, mud and marl ripples [41], I2 of rock, marl ripples and mud seafloors, I3 of mud, sand and marl ripples, I4 of marl and marl ripples and I5 of sand, sand ripples and rock. For I2 and I3, the angular variability of the sefloors especially marl ripples (for I2) and marl (for I3) is visually clear.

For all these images, we first determine the most discriminant features among the initial set of 219 features: we apply the algorithm described in Section II and detailed in [48] and we keep only the feature set such that the cumulative sum of weights exceeds 0.9 . Only a small number of features are retained. For example, figure 8 shows the plot of feature weights computed for image I1 and I5. For I1, the two co-occurrence matrices account for more than $90 \%$ of the total weight sum, these co-occurrence are computed for parameters $\left(d_{x}, d_{y}\right) \in\{(1,4),(2,1)\}$. For I5, the co-occurrence distributions computed for parameters $\left(d_{x}, d_{y}\right) \in\{(2,1),(2,2)\}$ are selected. For sonar images, we noticed that co-occurrence matrices are the most selected features. In previous work on Brodatz textures 


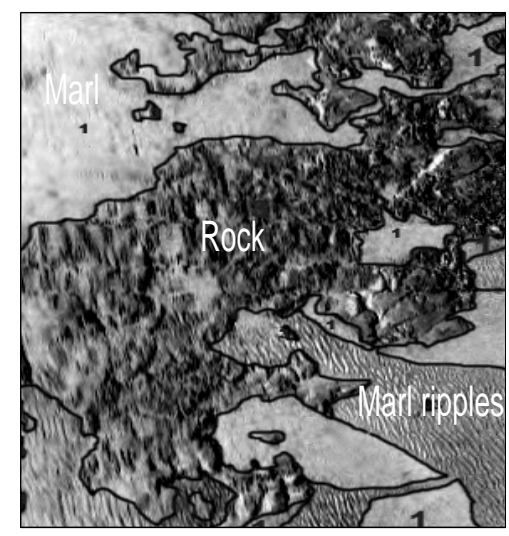

(I1)

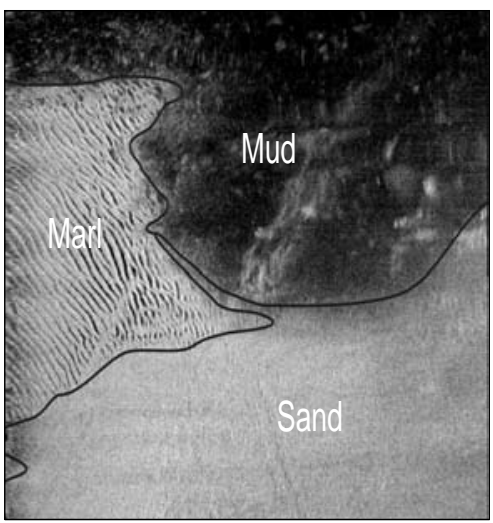

(I3)

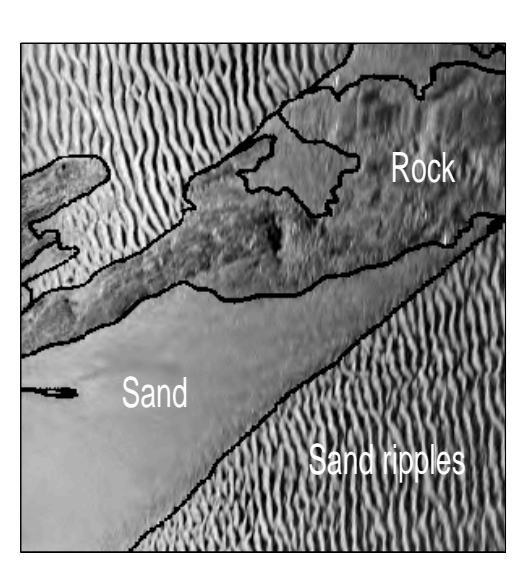

(I5)

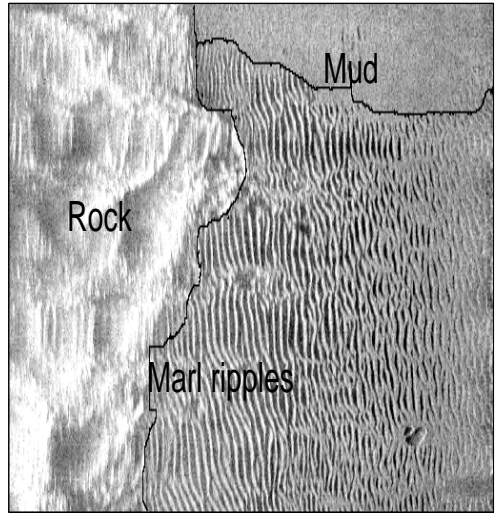

(I2)

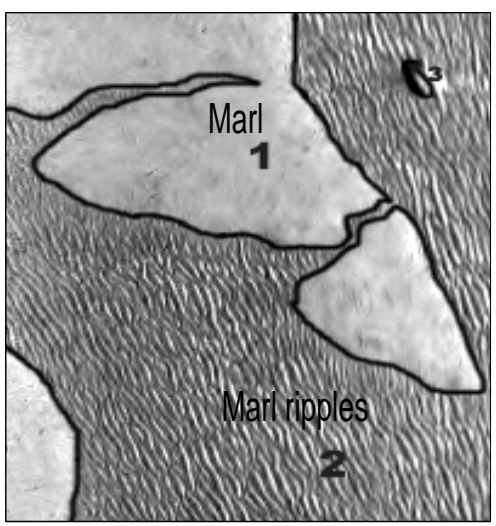

(I4)

Fig. 7. Test images and their manual segmentation (in black line). 


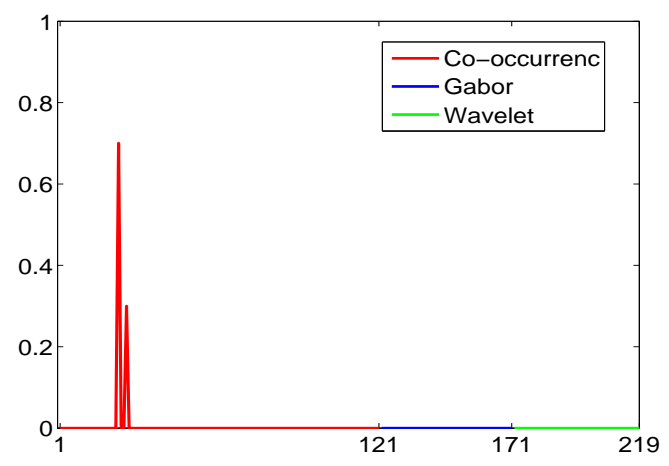

(a) I1

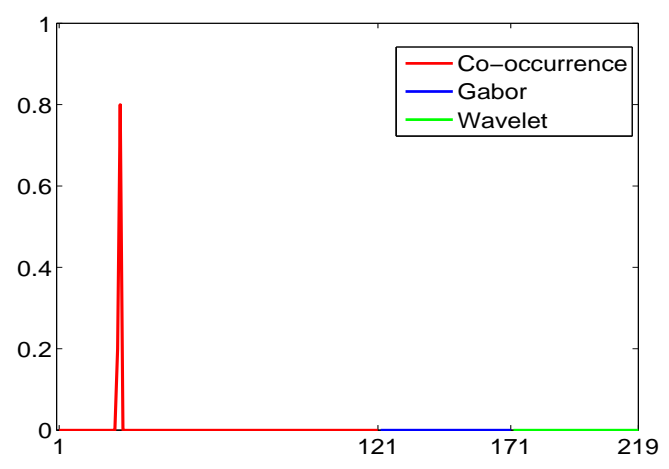

(b) $\mathrm{I5}$

Fig. 8. Feature weights: $1 \rightarrow 121$ co-occurrence distributions, $122 \rightarrow 171$ Gabor filter based features and $172 \rightarrow 219$ wavelet based distributions.

[48], [49], we remarked that Gabor and wavelet filters were selected for oriented textures whereas co-occurrence distributions, which in addition to the detection of texture structures detect the intensity values change, are selected in the case of texture having different intensity values and texture with regular motifs.

For the five test images, three segmentation algorithms are compared:

- The maximum likelihood segmentation denoted by ML. This method consists in maximizing at each pixel the conditional probability $p\left(y_{s} / x_{s}\right)$ given by equation (8):

$$
\hat{x_{s}}=\arg \max _{k} p\left(y_{s} / x_{s}=k\right)
$$

Several analysis window sizes are compared: $T_{W} \in\{7 \times 7,17 \times 17,33 \times 33\}$;

- The MMP segmentation described in Section III, applied for several analysis window sizes: $T_{W} \in\{7 \times 7,17 \times 17,33 \times 33\}$;

- The region-based variational segmentation described in Section IV.

Table I summarizes the different error classification rates for all segmentations. All segmentation methods give quite

\begin{tabular}{|c||c|c|c|c|c|c|c|}
\hline & ML: $T_{W}=7$ & ML: $T_{W}=17$ & ML: $T_{W}=33$ & MMP: $T_{W}=7$ & MMP: $T_{W}=17$ & MMP: $T_{W}=33$ & Variational \\
\hline I1 & $\tau=15 \%$ & $\tau=10 \%$ & $\tau=13.2 \%$ & $\tau=12.5 \%$ & $\tau=9.2 \%$ & $\tau=13 \%$ & $\tau=\mathbf{7 . 5} \%$ \\
\hline I2 & $\tau=15 \%$ & $\tau=11.5 \%$ & $\tau=7 \%$ & $\tau=10 \%$ & $\tau=4 \%$ & $\tau=5 \%$ & $\tau=\mathbf{3} \%$ \\
\hline I3 & $\tau=10 \%$ & $\tau=8 \%$ & $\tau=9 \%$ & $\tau=7 \%$ & $\tau=8 \%$ & $\tau=8 \%$ & $\tau=4 \%$ \\
\hline I4 & $\tau=5 \%$ & $\tau=4.5 \%$ & $\tau=6 \%$ & $\tau=4 \%$ & $\tau=4 \%$ & $\tau=6 \%$ & $\tau=\mathbf{3} \%$ \\
\hline I5 & $\tau=9.5 \%$ & $\tau=9 \%$ & $\tau=11 \%$ & $\tau=8 \%$ & $\tau=8 \%$ & $\tau=10 \%$ & $\tau=\mathbf{7} \%$ \\
\hline
\end{tabular}

TABLE I

SEGMENTATION ERROR RATES

good results according to the mean classification error rates. MMP and variational approaches are more efficient than 

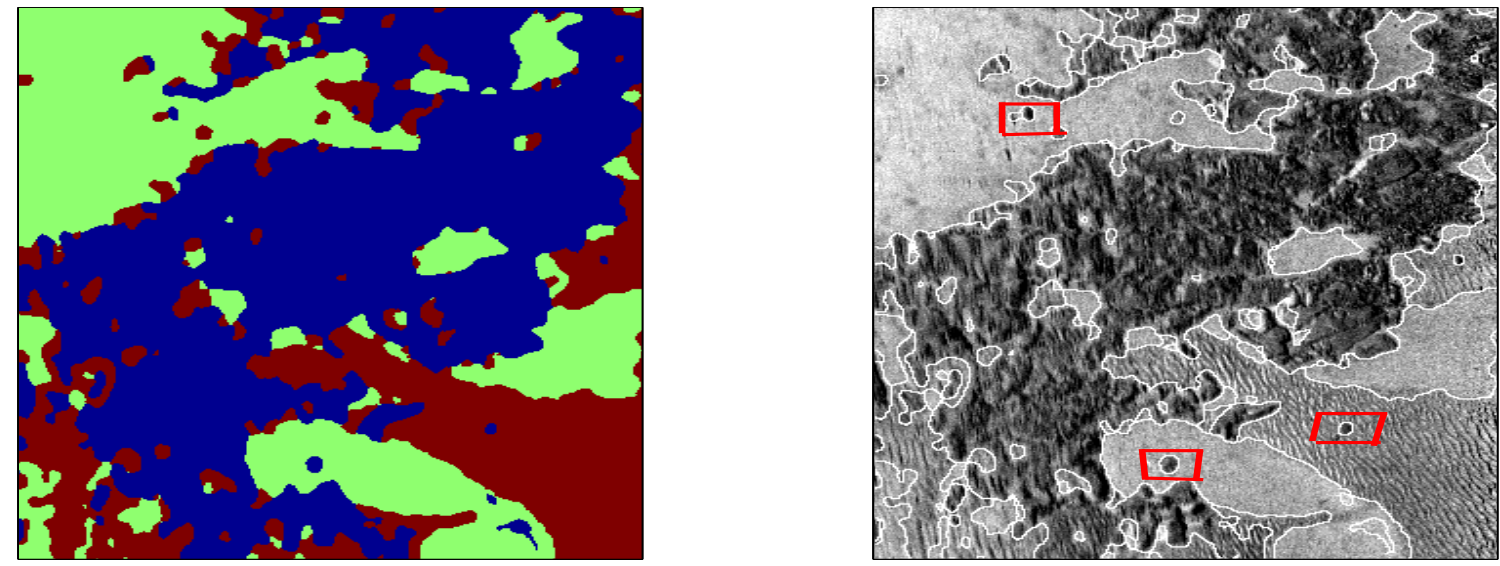

Fig. 9. MMP Segmentation of I1 using $T_{W}=7 \times 7, \tau=12.5 \%$.

the ML based segmentation because they take into account the spatial dependency between pixels. The difference between these later approaches (MMP and variational methods) mainly lies in the accuracy of the localization of region boundaries and in the dependency of MMP based segmentation on the window size $T_{W}$. In fact, for the MMP segmentation the use of of small neighborhood $\left(T_{W}=7 \times 7\right)$ leads to more accurate region frontiers but small misclassified patches appear because of the neighborhood inefficiency for texture characterization. Conversely, the use of large window sizes, $\left(T_{W}=33 \times 33\right)$ resorts to a lack of accuracy in the localization of the boundaries of the seabed regions because texture features extracted for pixels close to region boundaries involve a mixture of texture characteristics. The variational region-based approach does not need the choice of an analysis window and operates globally on region composed of pixels belonging to the same class. It resorts to a trade-off between segmentation accuracy and region homogeneity. Figures 9, 10, 11, 12 and 13 illustrate examples of the dependency of MMP segmentation on the sizes of analysis window and on the robustness of the variational approach. In figure 14, we plot the mean error rate for different window sizes.

We note that MMP and variational segmentations give similar results when the analysis window size is well chosen for instance, $T_{W}=17 \times 17$ for image I2 (figure 15), but its performance depends a lot on the choice of this parameter. This method can however be appropriate when the aim of the segmentation is to detect texture regions and without a seek of accurate boundaries.

The variational approach is also interesting because it is much faster than the MMP segmentation. Being deterministic, the variational approach, can be very fast especially if we use appropriate initialization such as an initial segmentation based on the Maximimum Likelihood criterion (ML). Whereas the MMP segmentation needs a large number of iterations, each iteration is also complex and involves Gibbs sampling. For our implementations the convergence time of a variational image typically corresponds to one iteration of the MMP algorithm.

To stress the interest of taking into account texture variability with respect to incidence angles, additional segmentation results are reported for image I 2 and I3 for witch the seafloor texture variability is more clear. In table 

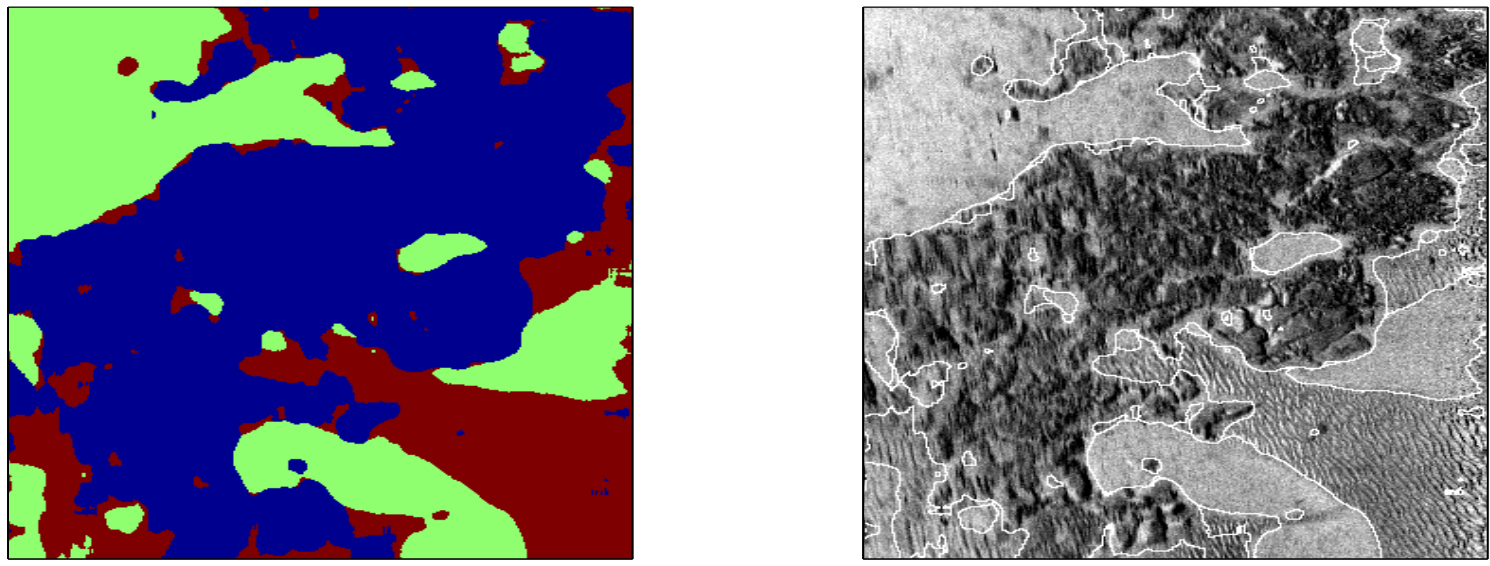

Fig. 10. MMP Segmentation of I1 using $T_{W}=17 \times 17, \tau=9.2 \%$.
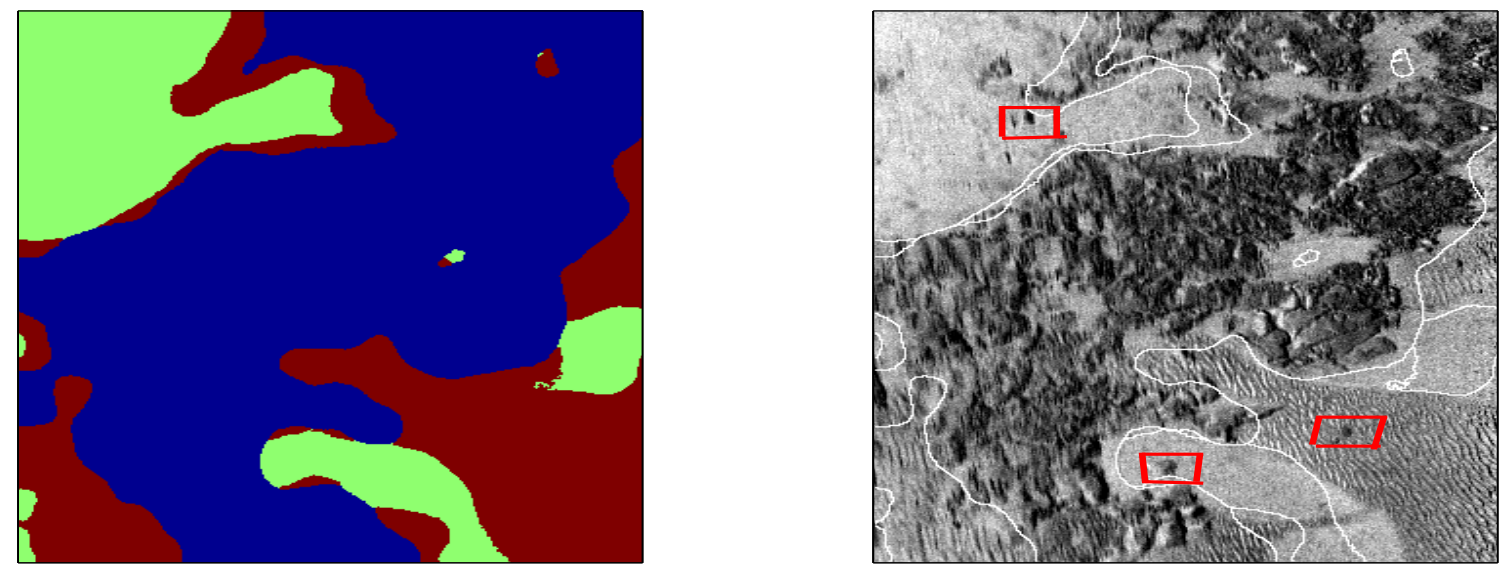

Fig. 11. MMP Segmentation of I1 using $T_{W}=33 \times 33, \tau=13 \%$.
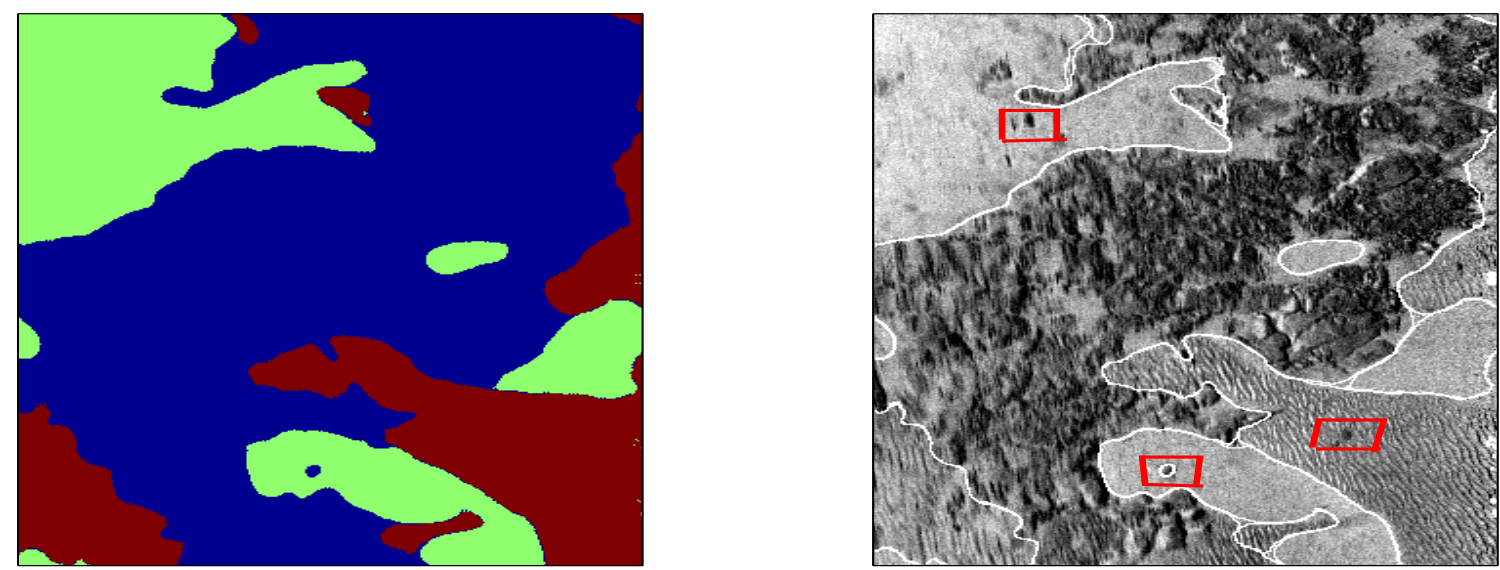

Fig. 12. Region based segmentation of $\mathrm{I} 1, \tau=7.5 \%$. 


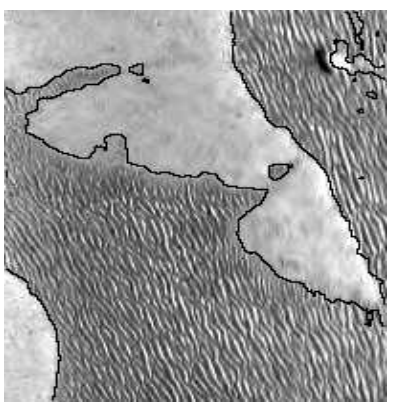

(a) MMP: $T_{W}=7, \tau=4 \%$

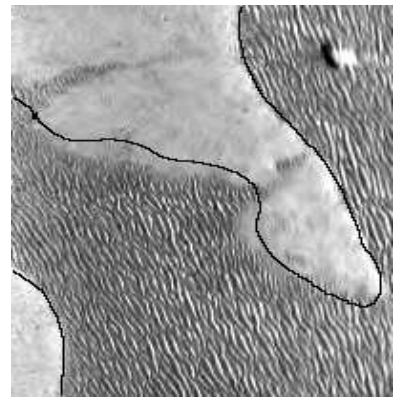

(b) MMP: $T_{W}=33, \tau=6 \%$

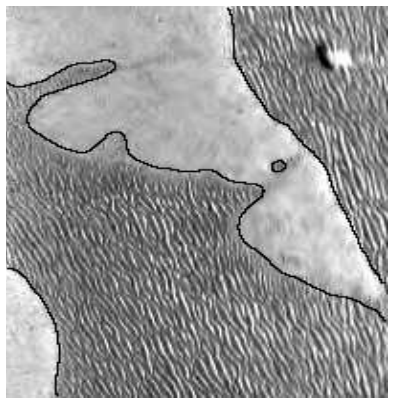

(c) Variational method, $\tau=3 \%$

Fig. 13. Segmentations of I4

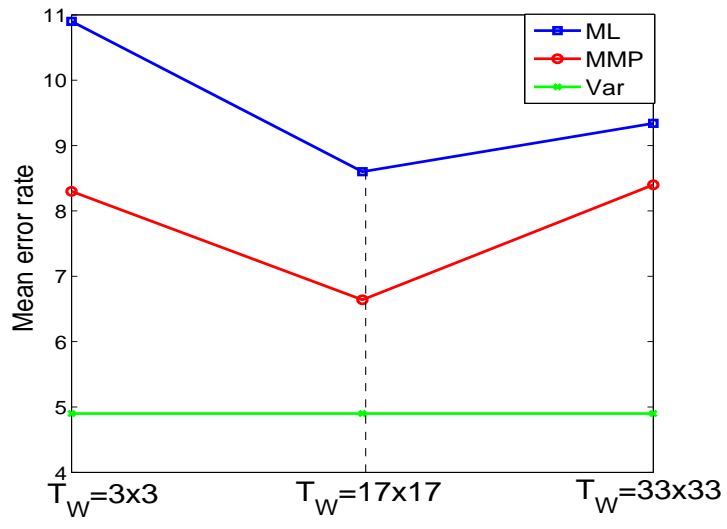

Fig. 14. Mean segmentation error rate for several window sizes.

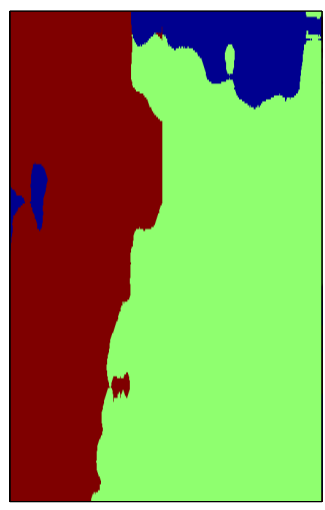

(a) MMP based segmentation $T_{W}=17, \tau=4 \%$
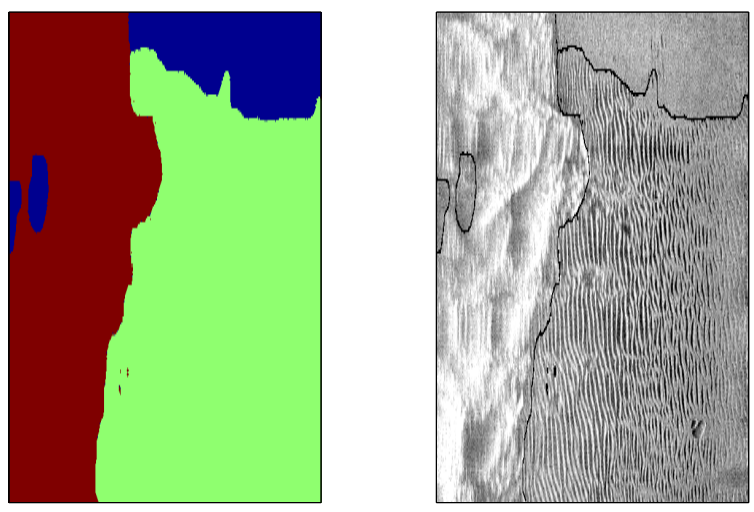

(b) Variationl based segmentation, $\tau=3 \%$

Fig. 15. I2 segmentations. 


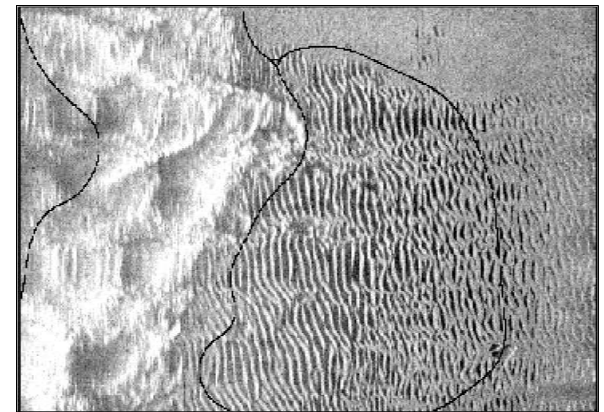

(a) Region based segmentation $J=1, \tau=23.5 \%$

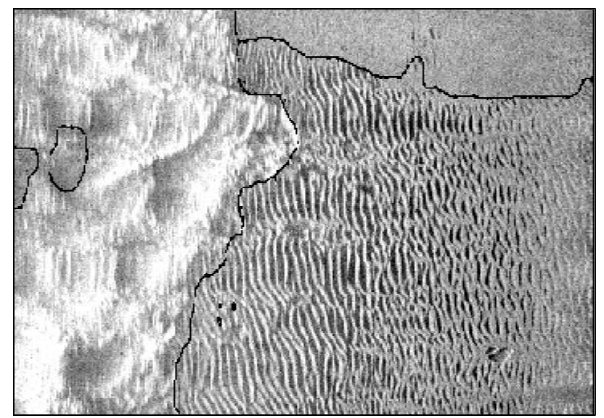

(b) Region based segmentation $J=3, \tau=3 \%$

Fig. 16. I2 Region based segmentation with and without angular weighting.

II, we summarize the segmentation error rates for the segmentation with $(\mathrm{J}=3)$ and with no angular weighting, i.e using only one angular domain $J=1$, for ML, MMP and variational methods. In figures 16 and 17 are reported the

\begin{tabular}{|c||c|c|c|c|c|c|}
\hline \multicolumn{1}{|c||}{} & \multicolumn{2}{c|}{ ML: $T_{W}=33$} & \multicolumn{2}{c|}{ MMP: $T_{W}=33$} & \multicolumn{2}{c|}{ Variational } \\
\hline & $J=1$ & $J=3$ & $J=1$ & $J=3$ & $J=1$ & $J=3$ \\
\hline I2 & $\tau=25 \%$ & $\tau=\mathbf{7} \%$ & $\tau=21 \%$ & $\tau=\mathbf{5} \%$ & $\tau=23.5 \%$ & $\tau=\mathbf{3} \%$ \\
\hline I3 & $\tau=20 \%$ & $\tau=\mathbf{9} \%$ & $\tau=16 \%$ & $\tau=\mathbf{8} \%$ & $\tau=15 \%$ & $\tau=\mathbf{4} \%$ \\
\hline
\end{tabular}

TABLE II

SEGMENTATION ERROR RATES

associated segmentation results. It can be noticed that a classical segmentation (without taking into account texture variability within incidence angles) cannot distinguish between visually similar seafloors (mud and marl ripples for I2 and marl and sand for I3) near the specular domain.

\section{CONCLUSION}

We proposed two segmentation algorithms for sonar image segmentation: a Bayesian algorithm using local statistics and a region based variational algorithm, both based on a novel similarity measure between seafloor type images in the feature space spanned by a large set of various texture statistics. This similarity measure is expressed as a weighted sum of Kullback-Leibler divergences between individual seafloor filter response statistics. The resulting weighting factors are exploited on the one hand for filter selection and, on the other hand, for taking into account the incidence angular dependency of seafloor textures. The conclusion is the co-occurrence matrices outperform the other features for our sonar images. The results show that the performance of the Bayesian approach depends on the size of analysis window. For pixel-based segmentation (maximum likelihood and MMP segmentations), the size must 


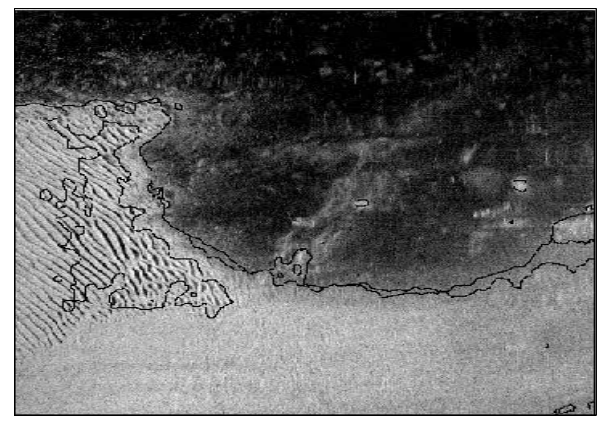

(a) MMP based segmentation $J=1, \tau=16 \%$

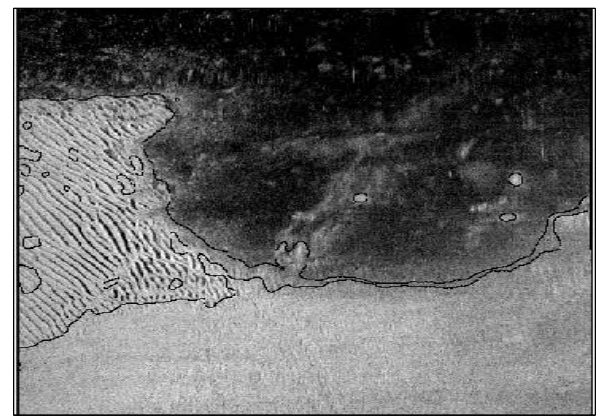

(b) MMP based segmentation $J=3, \tau=8 \%$

Fig. 17. I3 MMP based segmentation with and without angular weighting.

not be neither too large neither too small (according to the coarseness of given textures). The results also stress the suitability of the region based approach compared to the Bayesian pixel-based scheme for texture segmentation and the effectiveness of the proposed algorithm in taking into account the angular backscatter variabilities to discriminate between seafloor types especially near the specular sector.

\section{APPENDIX A}

\section{EVOLUTION EQUATION COMPUTATION}

Using the shape derivative tools, we want to differentiate the functional:

$$
F\left(\Omega_{k}\right)=K L_{w}^{\Theta}\left(Q^{k}, D\left(\Omega_{k}\right)\right)
$$

which can be written as follows:

$$
\sum_{j=1}^{J} \sum_{f=1}^{F} \pi_{\Omega_{k}}(j) w_{f}^{2} \int_{R_{f}} Q_{f, j}^{k}(\alpha) \log \left(\frac{Q_{f, j}^{k}(\alpha)}{D_{f, j}\left(\Omega_{k}, \alpha\right)}\right) d \alpha
$$

Let us introduce the following notations:

$$
\begin{aligned}
& \text { - } H_{j}^{k}=Q_{f, j}^{k}(\alpha) \log \left(\frac{Q_{f, j}^{k}(\alpha)}{D_{f, j}\left(\Omega_{k}, \alpha\right)}\right) . \\
& \text { - } G_{1}=\int_{\Omega_{k}} p\left(\Theta_{j} / z_{s}\right) g_{\sigma_{f}}\left(h_{f}(s)-\alpha\right) d s .
\end{aligned}
$$

Then, we have:

$$
F\left(\Omega_{k}\right)=\sum_{j=1}^{J} \sum_{f=1}^{F} w_{f}^{2} \pi_{\Omega_{k}}(j) \int_{R_{f}} H_{j}^{k}
$$

The Gâteaux derivative of $F\left(\Omega_{k}\right)$ in the direction of a vector field $\vec{V}$ is given by:

$$
d F\left(\Omega_{k}, \vec{V}\right)=\sum_{j=1}^{J} \sum_{f=1}^{F} w_{f}^{2} d \pi_{\Omega_{k}}(j)\left(\Omega_{k}, \vec{V}\right) \int_{R_{f}} H_{j}^{k} d \alpha+\pi_{\Omega_{k}}(j) \int_{R_{f}} d H_{j}^{k}\left(\Omega_{k}, \vec{V}\right) d \alpha
$$


Theorem (see [37])

The Gâteaux derivative of a functional of the type $K(\Omega)=\int_{\Omega} k(s, \Omega) d s$ is given by:

$$
d K(\Omega, \vec{V})=\int_{\Omega} k_{s h}(s, \Omega, \vec{V}) d s-\int_{\Gamma} k(s, \Omega)(\vec{V} \vec{N}) d a(s)
$$

where $\Omega$ denoted a region and $\Gamma$ its boundary, $k_{s h}(s, \Omega, \vec{V})$ is the shape derivative of $k(s, \Omega)$.

In our case:

$$
d \pi_{\Omega_{k}}(j)\left(\Omega_{k}, \vec{V}\right)=-\int_{\Gamma_{k}} p\left(\Theta_{j} / z_{s}\right)\left(\vec{V} \cdot \vec{N}_{k}\right) d \vec{a}(s)
$$

$H_{j}^{k}$ can be written as:

$$
H_{j}^{k}=Q_{f, j}^{k}(\alpha) \log \left(\frac{\pi_{\Omega_{k}}(j) Q_{f, j}^{k}(\alpha)}{G_{1}\left(\Omega_{k}, f, j, \alpha\right)}\right)
$$

Using the chain rule, we get:

$$
\begin{gathered}
d H_{j}^{k}\left(\Omega_{k}, \vec{V}\right)=\left(\frac{\partial H_{j}^{k}}{\partial G_{1}} d G_{1}\left(\Omega_{k}, \vec{V}\right)+\frac{\partial H_{j}^{k}}{\partial \pi_{\Omega_{k}}(j)} d \pi_{\Omega_{k}}(j)\left(\Omega_{k}, \vec{V}\right)\right) \\
\frac{\partial H_{j}^{k}}{\partial G_{1}}=-\frac{Q_{f, j}^{k}}{G_{1}} \\
\frac{\partial H_{j}^{k}}{\partial \pi_{\Omega_{k}}(j)}=\frac{Q_{f, j}^{k}}{\pi_{\Omega_{k}}(j)}
\end{gathered}
$$

According to the previous theorem, we get:

$$
d G_{1}\left(\Omega_{k}, \vec{V}\right)=-\int_{\Gamma_{k}} p\left(\Theta_{j} / z_{s}\right) g_{\sigma_{f}}\left(h_{f}(s)-\alpha\right)\left(\vec{V} \cdot \vec{N}_{k}\right) d \vec{a}(s)
$$

Finally, we get:

$$
\begin{aligned}
& d H_{j}^{k}\left(\Omega_{k}, \vec{V}\right) \\
&=\int_{\Gamma_{k}} p\left(\Theta_{j} / z_{s}\right)\left[\frac{Q_{f, j}^{k}(\alpha)}{G_{1}\left(f, \alpha, \Omega_{k}\right)} g_{\sigma_{f}}\left(h_{f}(s)-\alpha\right)-\frac{Q_{f}(\alpha)}{\pi_{\Omega_{k}}(j)}\right]\left(\vec{V} \cdot \vec{N}_{k}\right) d \vec{a}(s) \\
&=\frac{1}{\pi_{\Omega_{k}}(j)} \int_{\Gamma_{k}} p\left(\Theta_{j} / z_{s}\right)\left[\frac{Q_{f, j}^{k}(\alpha)}{D_{f, j}\left(\Omega_{k}, \alpha\right)} g_{\sigma_{f}}\left(h_{f}(s)-\alpha\right)-Q_{f}(\alpha)\right]\left(\vec{V} \cdot \vec{N}_{k}\right) d \vec{a}(s) \\
& \int_{R_{f}} d H_{j}^{k}\left(\Omega_{k}, \vec{V}\right) d \alpha=\frac{1}{\pi_{\Omega_{k}}(j)} \int_{\Gamma_{k}} p\left(\Theta_{j} / z_{s}\right)\left[\frac{Q_{f, j}^{k}}{D_{f, j}\left(\Omega_{k}\right)} * g_{\sigma_{f}}\left(h_{f}(s)\right)-1\right]\left(\vec{V} \cdot N_{k}\right) d \vec{a}(s)
\end{aligned}
$$

Finally, according to equation 24:

$$
d F\left(\Omega_{k}, \vec{V}\right)=-\sum_{j=1}^{J} \sum_{f=1}^{F} p\left(\Theta_{j} / z_{s}\right) w_{f}^{2} \int_{\Gamma_{k}}\left[K L\left(Q_{f, j}^{k}, D_{f, j}\right)-\left(\frac{Q_{f, j}^{k}}{D_{f, j}\left(\Omega_{k}\right)}-1\right) * g_{\sigma_{f}}\left(h_{f}(s)\right)\right]\left(\vec{V} \cdot \vec{N}_{k}\right) d \vec{a}(s)
$$




\section{REFERENCES}

[1] P. Brehmer, F. Gerlotto, J. Guillard, F. Sanguinède, Y. Guénnegan and D. Buestel, "New applications of hydroacoustic methods for monitoring shallow water aquatic ecosystems: the case of mussel culture grounds", Aquatic Living Resources, vol.16, pp: 333-338, 2003.

[2] M. Mignotte, C. Collet, P. Perez and P. Bouthemy, "Hybrid genetic optimization and statistical model based approach for the classification of shadow shapes in sonar imagery", IEEE Transaction on Pattern Analysis and Machine Intelligence, vol.22, no. 2, pp: 129-141, Feb. 2000.

[3] L. Hellequin, J.M. Boucher and X. Lurton, "Processing of high-frequency multibeam echo sounder data for seafloor characterization", IEEE Journal of Oceanic Engineering, vol.28, no.1, pp: 78-89, Jan. 2003.

[4] G.Le. Chenadec and J.M. Boucher, "Sonar image segmentation using the angular dependence of backscattering distributions", Oceans Europe, vol.1, pp: 147-152, Brest, 2005.

[5] G. Le Chenadec and Jean-Marc Boucher and X. Lurton, "Angular dependency of K-distributed sonar data", IEEE on Geoscience and Remote Sensing, vol.45, no.5, pp: 1224-1236, May, 2007.

[6] E. Jakeman, "Non gaussian models for the statistics of scattered waves", Advances in Physics, vol.37, no.5, pp: 471-529, 1988.

[7] A.P. Lyons et D.A. Abraham, "Statistical characterization of high-frequency shallow-water sea-floor backscatter", Journal of the Acoustical Society of America, vol.106, no.3, pp: 1307-1315, 1999.

[8] D.A. Abraham and A.P. Lyons, "Novel physical interpretations of k-distributed reverberation", IEEE Journal of Oceanic Engineering, vol.27, no.4, pp: 800-813, Oct, 2002.

[9] NC. Mitchell, "Processing and analysis of Simrad multibeam sonar data", Marine Geophysical Researches. Vol.18, pp: 729-739, 1996.

[10] G.Le. Chenadec and J.M. Boucher and X. Lurton and J.M. Augustin, "Angular dependence of statistical distributions for backscattered signals: modeling and application to multibeam echosounder data", Oceans, vol.2, pp: 897-903, Sept, 2003.

[11] D.R. Jackson and D.P. Winbrenner and A. Ishimaru, "Application of the composite roughness model to high-frequency bottom backscattering", The Journal of the Acoustical Society of America, vol.79, no.5, pp: 1410-1422, 1986.

[12] C. de Moustier and D. Alexandrou, "Angular dependence of $12 \mathrm{kHz}$ seafloor acoustic backscatter", The Journal of the Acoustical Society of America, vol.90, no.1, pp: 522-531, 1991.

[13] J.E. Hughes-Clarke, "Toward remote seafloor classification using the angular response of acoustic backscatter: a case study from multiple overlapping GLORIA Data”, IEEE Journal of Oceanic Engineering, vol.19, no.1, pp: 112-127, Jan, 1994.

[14] E. Pouliquen and X. Lurton, "Automated Sea-bed Classification System For Echo-Sounders", Oceans, vol.1, pp: 317-321, Oct, 1992.

[15] D. Langer and M. Herbert, "Building qualitative elevation maps from side scan sonar data for autonomous underwater navigation", Proc. IEEE International Conference on Robotics and automation, vol.3, pp: 2478-2483, Apr, 1991.

[16] R. Li and S. Pai, "Improvement of bathymetric data bases by shape from shading technique using side-scan sonar images", OCEANS, vol.1, pp: 320-324, Oct, 1991.

[17] E. Dura and J. Bell and D. Lane, "Reconstruction of textured seafloors from side-scan sonar images", IEE Proc. Radar Sonar Navigation, vol.151, no.2, pp: 114-125, Apr, 2004.

[18] A.E. Johnson and M. Hebert, "Seafloor map generation for autonomous underwater vehicle navigation", Autonomous Robots, vol.3, no.2, pp: 145-168, 1996.

[19] R. Zhang and P. Tsai and J.E. Cryer and M. Shah, "Shape from shading: a survey", IEEE Transaction on Pattern Analysis and Machine Intelligence, vol.21, no.8, pp: 690-705, Aug, 1999.

[20] T.H. Eggen, "Acoustic sediment classification experiment by means of multibeam echosounders", Proc. Acoustic Classification and Mapping of Seabed, vol.15, no.2, pp: 437-444, 1993.

[21] L.M. Linnett and S.J. Clarke and D.R. Carmichael, "The analysis of sidescan sonar images for seabed types and objects", Proc. Second European Conference on Underwater Acoustics, vol.22, pp: 187-194, 1994.

[22] M. Lianantonakis and Y.R. Petillot, "Sidescan sonar segmentation using active contours and level set method", OCEANS- Europe, vol.1, pp: 719-724, Brest, 2005.

[23] L. Xiuwen et W. DeLiang, “Texture Classification Using Spectral Histograms”, IEEE Transactions on Image Processing, vol.12, no.6, pp: 661-670, June, 2003.

[24] O.G. Cula and K. Dana, "3D Texture Recognition Using Bidirectional Feature Histograms”, International Journal of Computer Vision,vol.59, no.1, pp: 33-60, Aug, 2004. 
[25] R. Fablet and P. Bouthemy, "Motion recognition using non parametric image motion models estimated from temporal and multiscale cooccurrence statistics", IEEE Transactions Pattern Analysis and Machine Intelligence, vol.25, no.12, pp: 1619-1624, Dec, 2003.

[26] P. Nammalwar, O. Ghita and P.F. Whelan, "Integration of feature distributions for color texture segmentation", International Conference on Pattern Recognition, vol.1, pp: 716-719, Aug, 2005.

[27] Q. Xu, J. Yang and S. Ding, "Texture Segmentation using LBP embedded Region Competition", Electronic Letters on Computer Vision and Image Analysis, vol.5, no.1, pp: 41-47, 2004.

[28] S. Kullback, "On information and sufficiency”, The Annals of Mathematical Statistics, vol.22, Wiley, New York, 1951.

[29] E. Parzen, “On the Estimation of a Probability Density Function and the Mode", Annals of Mathematical Statistics, vol.33, pp: 1065-1076, 1962.

[30] R.M. Haralick, "Statistical and Structural Approaches to Texture”, Proceedings of the IEEE, vol.67, no.5, pp: 786-804, 1979.

[31] J. Marroquin, S. Mitter and T. Poggio, "Probabilistic solution of ill-posed problems in computational vision", Journal of the American Statistical Association, vol.82, pp: 76-89, 1987.

[32] M. Kass, A. Withkin et D. Terzopoulos, "Snakes: Active contour models", International Journal of Computer Vision, vol.1, no.4, pp: 321-331, 1988.

[33] J.A. Sethian, "Level set methods", Combridge University Press, 1996.

[34] H.K. Zhao, T. Chan, B. Merriman et S. Osher, "A variational level set approach to multiphase motion”, J. Comp. Phy., vol.127, pp: 179-19, 1996.

[35] J.F Aujol, G. Aubert and L. Blanc-Féraud, "Wavelet-based level set evolution for classification of textured images". IEEE Transaction on Image Processing, vol. 12, no.12. pp: 1634-1641, Dec, 2003.

[36] C. Samson, L. Blanc-Féraud, G. Aubert and J. Zerubia, “A level set method for image classification”. International Journal on Computer Vision, vol.40, no.3. pp: 187-197, 2000.

[37] S. Jehan-Besson, M. Barlaud et G. Aubert, "Image segmentation using active contours: calculus of variations or shape gradients?", SIAM J. APPL. MATH, vol.63, no.6, pp: 2128-2154, 2003.

[38] H.K. Zhao, T. Chan, B. Merriman and S. Osher, "A variational level set approach to multiphase motion". J. Comp. Phy.,vol.127, pp: 179-195, 1996.

[39] A. Luminita, A. Vese and T.F. Chan, "A multiphase level set framework for image segmentation using the Mumford and Shah model". Int. Journal of Comp. Vis. ,vol.50, no.3, pp: 271-293, 2002.

[40] I. Karoui, R. Fablet, J.M. Boucher and J.M. Augustin, "Separability-Based Kullback Divergence Weighting and Filter Selection for Texture Classification and Segmentation”. 18th Int. Conf. on Pattern Recognition, ICPR, Hong-Kong, Aug, 2006.

[41] A. Ehrhold and D. Hamon and B. Guillaumont, "The Rebent monitoring network, a spatial integrated acoustic approach to survey nearshore macrobenthic habitats: application to the bay of Concarneau (South Brittany, France)", ICES Journal of Marine Science,vol.63, pp: 1604$1615,2006$.

[42] S. Geman and G. Geman, "Stochastic relaxation, Gibbs distributions and the Bayesian restoration of images". IEEE Transaction on Pattern Analysis and Machine Intelligence, vol.6, no.6, pp: 721-741, 1984.

[43] W. Pieczynski, (1992) Statistical image segmentation. Machine Graphics and Vision. Vol.1, no.2, pp: 261-268.

[44] A.P. Lyons et D.A. Abraham, "Statistical characterization of high-frequency shallow-water sea-floor backscatter", Journal of the Acoustical Society of America, Vol.106, no.3, pp.1307-1315, 1999.

[45] D.A. Abraham et A.P. Lyons, "Novel physical interpretations of k-distributed reverberation", IEEE Journal of Oceanic Engineering, Vol.27, no.4, pp.800-813, 2002.

[46] L. Hellequin, J.M. Boucher et X. Lurton, "Processing of high-frequency multibeam echo sounder data for seafloor characterization", IEEE Journal of Oceanic Engineering, Vol.28, no.1, pp.78-89, 2003.

[47] G.Le. Chenadec et J.M. Boucher, "Sonar image segmentation using the angular dependence of backscattering distributions", Oceans 2005 - Europe, Vol.1, pp.147-152, 2005.

[48] I. Karoui, R. Fablet, J.M. Boucher and J.M. Augustin, "Separability-Based Kullback Divergence Weighting and Filter Selection for Texture Classification and Segmentation”. 18th Int. Conf. on Pattern Recognition, ICPR, Hong-Kong, Aug, 2006.

[49] I. Karoui, R. Fablet, J.M. Boucher, W. Pieczynski and J.M. Augustin, "Fusion of textural statistics using a similarity measure: application to texture recognition and segmentation”. Pattern analysis and applications, Computer Science, 2008. 